\title{
Convective Ripening and Initiation of Rainfall
}

\author{
Michael Wilkinson \\ Department of Mathematics and Statistics, The Open University, Walton Hall, Milton Keynes, MK7 6AA, England
}

PACS 92.60.Nv - Cloud physics and chemistry

PACS 92.60.hk - Convection, turbulence, and diffusion

PACS 92.60.Mt - Particles and aerosols

\begin{abstract}
This paper discusses the evolution of the droplet size distribution for a liquid-in-gas aerosol contained in a Rayleigh-Bénard cell. It introduces a non-collisional model for broadening the droplet size distribution, termed 'convective ripening'. The paper also considers the initiation of rainfall from ice-free cumulus clouds. It is argued that while collisional mechanisms cannot explain the production of rain from clouds with water droplet diameters of $20 \mu \mathrm{m}$, the noncollisional convective ripening mechanism gives a much faster route to increasing the size of the small fraction of droplets that grow into raindrops.
\end{abstract}

Introduction. - The dynamics of the onset of rain' fall from ice-free ('warm') cumulus clouds is poorly understood [1]. Coalescence of droplets which collide due to differential rates of gravitational settling is effective for ' droplets with radius $a$ above $50 \mu \mathrm{m}$, and leads to a runaway growth to produce millimetre-scale raindrops [5]. Many clouds are found to contain droplets with radius approximately $10-15 \mu \mathrm{m}$, which result from primary condensation onto aerosol nuclei. For droplets in this size range, growth by collisional coalescence is slow because the collision rates and the collision efficiencies are low [1]. This makes it difficult to explain observations of the rapid onset ' of rainfall from warm cumulus clouds. (Rainfall from icebearing clouds is easier to explain: see [1] for a discussion ' of the Bergeron process).

It is, therefore, desirable to formulate models for non-collisional growth of water droplets, in which some droplets are able to grow at the expense of others shrinking, by transferring water molecules between droplets as water vapour. Ostwald ripening [6] is one such mechanism, but it is too slow to be significant in terrestrial clouds [7, while it is relevant to test-tube models for rainfall [8]. It has been suggested that condensation processes may be able to cause the droplet size distribution to broaden due to fluctuations in the degree of supersaturation. This possibility has been addressed by numerous authors: see, for example, 9 17. These investigations have used numerical simulations, and it is difficult to draw conclusions which are applicable to real clouds because of the limited range of size scales which can be simulated reliably. The models which are used in these studies also have a large number of parameters. These factors make it difficult to obtain general conclusions.

This work will consider a benchmark model for the broadening of the droplet size distribution of an aerosol due to convection. This process will be termed 'convective ripening' to distinguish it from Ostwald ripening. This work considers how the process works in the simplest relevant model, which is an aerosol in a Rayleigh-Bénard convection cell. As well as having fewer physical parameters than a cloud, this system can be subject to a carefully controlled laboratory investigation. It would, however, be very difficult to perform a numerical simulation of this system in the parameter range describing clouds, because the important physical processes involve all lengthscales of the system.

Having described a non-collisional model for droplet growth, this will be applied to rain initiation from icefree ('warm') cumulus clouds. An important aspect of this problem is that the conversion of a microscopic water droplet into a rain droplet is a very rare event (this fact was previously emphasised by Kostinski and Shaw [5]). However, the growth of a droplet to the stage where runaway growth occurs is a multi-stage process. It is argued that the probability for the required number of favourable events to be achieved by collisional processes alone is extremely small. It is shown that droplets can grow much more rapidly the convective ripening mechanism.

This paper complements a recent work which discussed Ostwald ripening as a non-collisional model for the evolu- 
tion of the droplet size distribution [8]. That work concluded that while Ostwald ripening provides the correct description of a test-tube model for rainfall, it acts too slowly to explain rain from warm cumulus clouds.

Discussions of convection processes within clouds often involve the complex and poorly understood issue of 'entrainment' of air into a cloud (see, for example [13, 17]). This paper argues that a Rayleigh-Bénard cell appears to be a sufficient model to understand the mechanism of rainfall from warm cumulus clouds. Because entrainment does not enter into the Rayleigh-Bénard system which is discussed in this paper, hypotheses about entrainment are not a necessary feature of understanding rainfall from warm cumulus clouds.

The convection cell model. - Consider a RayleighBénard convection cell, in which the working fluid is a gas (air, say) containing an aerosol suspension of liquid droplets (water, say). The height of the cell is $h$ and the temperature difference between the upper and lower plates is $\Delta T_{h}$. It is assumed that the horizontal dimensions of the cell are large compared to $h$. The rate of heat transfer per unit area is $Q$. The gas has volume-specific heat capacity at constant pressure $C_{\mathrm{g}}$, density $\rho_{\mathrm{g}}$, kinematic viscosity $\nu$ and thermal diffusivity is $D_{\text {th }}$. It will be assumed the convection in the container is in a turbulent regime, with rate of dissipation per unit mass $\epsilon$.

The cell contains an aerosol of liquid droplets with density $\rho_{1}$ and volume-specific heat of evaporation $L$. The vapour of the aerosol liquid in the carrier gas has diffusivity $D$. The number density of droplets is $n_{0}$, and the probability density function for the droplet radius $a$ at time $t$ is $P(a, t)$.

The objective is to understand how convection affects the distribution of sizes of the aerosol droplets. It will be assumed that the rate of collisions between the droplets is negligible. It will also be assumed that collisions of aerosol droplets with the walls of the container is not a significant process. The validity of this assumption is not critical to using this system as a model for cloud physics (because there is no material container in that context).

In order to understand the ripening of the droplet size distribution it is necessary to consider first how the aerosols responds to changes in the temperature of the surrounding gas, and then how the convection process influences the temperature.

\section{Response to temperature fluctuations. -} Changes in the temperature of the surrounding gas cause the size of the droplets to change due to condensation or evaporation. This is characterised by two parameters, $T_{0}$ and $\tau_{\text {eq }}$, which describe, respectively, the sensitivity and the timescale of the response. It will be shown that if the majority of the aerosol droplets have radius close to $a_{0}$, the change $\delta a_{0}$ of the equilibrium radius in response to a temperature increment $\delta T$ satisfies

$$
\frac{\delta a_{0}}{a_{0}}=-\frac{\delta T}{T_{0}}
$$

to leading order in $\delta T$, and that the change in droplet radius occurs on a timescale $\tau_{\text {eq }}$. In the following expressions for both $T_{0}$ and $\tau_{\text {eq }}$ are obtained. Equivalent calculations can be found in many earlier works (reviewed in 1-4), but with differences in physical motivation an notation. A brief derivation is given here to make this paper unambiguous and self-contained.

The volume fraction of water molecules in the gas, $\Phi$, may be assumed to be uniform throughout the container because the system is well mixed by convection. This is the sum of contributions from water in the liquid and the vapour phase:

$$
\Phi=\Phi_{1}+\Phi_{\mathrm{v}} .
$$

The equilibrium vapour content above a flat liquid surface at temperature $T$ is denoted by $\Phi_{\text {eq }}(T)$, and there may be a degree of supersaturation, denoted by $s$. It is assumed that the droplets are sufficiently large that curvature and hygroscopic effects of the aerosol condensation nuclei can be neglected, so that the vapour mole fraction in the bulk of the gas phase will be written

$$
\Phi_{\mathrm{v}}=\Phi_{\mathrm{eq}}(T)+s .
$$

The volume-fraction of the liquid phase is

$$
\Phi_{1}=\frac{4 \pi}{3} n_{0}\left\langle a^{3}\right\rangle
$$

(throughout this paper $\langle X\rangle$ denotes the expectation value of any quantity $X$ ). If the temperature of the gas changes, the sizes of the droplets will change. For example, a decrease of the temperature results in a supersaturation which causes condensation on the surface of the droplets. The rate of the condensation process is determined by diffusion of vapour. The radius of a droplet changes at a rate

$$
\frac{\mathrm{d} a}{\mathrm{~d} t}=j_{\mathrm{v}}=-\frac{D}{a} \Delta \Phi_{\mathrm{v}}
$$

where $j_{\mathrm{v}}$ is the volume flux density of condensing molecules and $\Delta \Phi_{\mathrm{v}}$ is the the volume-fraction on the surface of the droplet minus the volume-fraction in the bulk of the gas phase. The surface of the droplet is in quasi-static equilibrium with the surrounding fluid, so there is no supersaturation at the surface. However, the temperature of the liquid droplet may be increased by an amount $\Delta T$ due to the latent heat of water condensing on the surface, so that

$$
\Delta \Phi_{\mathrm{v}}=\frac{\mathrm{d} \Phi_{\mathrm{eq}}}{\mathrm{d} T} \Delta T-s .
$$

The thermal flux density due to the latent heat is

$$
j_{\mathrm{th}}=-L \frac{\mathrm{d} a}{\mathrm{~d} t}=-\frac{D_{\mathrm{th}} C_{\mathrm{g}}}{a} \Delta T .
$$


Combining (6) and (7) gives

$$
\frac{\mathrm{d} a}{\mathrm{~d} t}=\frac{D}{a} s-\frac{D}{a} \frac{\mathrm{d} \Phi_{\mathrm{eq}}}{\mathrm{d} T} \frac{L a}{D_{\mathrm{th}} C_{\mathrm{g}}} \frac{\mathrm{d} a}{\mathrm{~d} T} .
$$

This gives a simple expression relating the rate of droplet growth to the supersaturation

$$
\frac{\mathrm{d} a}{\mathrm{~d} t}=\frac{D_{\mathrm{eff}}}{a} s
$$

where the effective diffusion constant is

$$
D_{\text {eff }}=\frac{D}{1+\Theta}, \quad \Theta=\frac{D L}{D_{\mathrm{th}} C_{\mathrm{g}}} \frac{\mathrm{d} \Phi_{\mathrm{eq}}}{\mathrm{d} T} .
$$

This treatment neglected the possibility of cross-coupling between thermal and mass fluxes (the Soret effect). This could be incorporated with a simple modification of the theory, but the coefficients of the off-diagonal terms of the transport matrix do not appear to have been definitively determined.

In the case where the temperature of the system varies extremely slowly, the supersaturation is always negligible, and the relation between droplet size and temperature is determined by writing

$$
\Phi=\frac{4 \pi}{3} n_{0} a_{0}^{3}(T)+\Phi_{\mathrm{eq}}(T)
$$

so that a small change in temperature $\delta T$ results in a small change of radius $\delta a_{0}$ given by (1), with coefficient

$$
T_{0}=3 \Phi_{1}\left(\frac{\mathrm{d} \Phi_{\mathrm{eq}}}{\mathrm{d} T}\right)^{-1} .
$$

Now consider the effect of varying the temperature of the aerosol at a finite rate, but still assuming that it is spatially homogeneous: write $T=T_{0}+\delta T(t), a=a_{0}\left(T_{0}\right)+\delta a(t)$, so that

$$
\Phi=\frac{4 \pi}{3} n_{0}\left(a_{0}+\delta a\right)^{3}+\Phi_{\mathrm{eq}}\left(T_{0}\right)+\frac{\mathrm{d} \Phi_{\mathrm{eq}}}{\mathrm{d} T} \delta T(t)+s .
$$

Taking the leading order in the small fluctuation $\delta a$ and using (9) yields the following equation for the response of the droplets to fluctuations in temperature:

$$
\frac{\mathrm{d} \delta a}{\mathrm{~d} t}=-\frac{1}{\tau_{\mathrm{eq}}} \delta a-\frac{a_{0}}{\tau_{\mathrm{eq}} T_{0}} \delta T(t)
$$

where the relaxation time is

$$
\tau_{\mathrm{eq}}=\frac{a_{0}^{2}}{3 \Phi_{1} D_{\mathrm{eff}}}=\frac{1}{4 \pi n_{0} a_{0} D_{\mathrm{eff}}} .
$$

At this stage it is relevant to make some estimates of the parameters $T_{0}$ and $\tau_{\text {eq }}$. The rate of change of the saturation volume-fraction is obtained from the ClausiusClapeyron relation, $\mathrm{d} p / \mathrm{d} T=L / T \Delta V$, where $\Delta V$ is the volume change on a phase transition. Assuming that the vapour pressure is sufficiently low that the ideal gas law is applicable,

$$
p=\frac{R T}{V_{\mathrm{m}}} \Phi_{\mathrm{eq}},
$$

where $V_{\mathrm{m}}$ is the molar volume of the liquid. Also, the volume change per mole associated with the phase transition is $\Delta V=V_{\mathrm{m}} / \Phi_{\text {eq }}$. The Clausius-Clapeyron equation can therefore be written in the form

$$
\frac{\mathrm{d} p}{\mathrm{~d} T}=\frac{L}{T} \Phi_{\mathrm{eq}} .
$$

Comparing (16) and (17) yields an expression for $\mathrm{d} \Phi_{\text {eq }} / \mathrm{d} T$, and hence

$$
T_{0}=\frac{3 R T}{L V_{\mathrm{m}}-R T} \frac{\Phi_{1}}{\Phi_{\mathrm{eq}}} T .
$$

For water at $T=278 \mathrm{~K}, L=2.4 \times 10^{9} \mathrm{~J} \mathrm{~m}^{-3}, C_{\mathrm{g}}=$ $800 \mathrm{Jm}^{-3}, V_{\mathrm{m}}=1.8 \times 10^{-5} \mathrm{~m}^{3} \mathrm{~mol}^{-1}, \Phi_{\mathrm{eq}}=7 \times 10^{-6}$, $D=2.5 \times 10^{-5} \mathrm{~m}^{2} \mathrm{~s}^{-1}$ and $D_{\text {th }}=1.9 \times 10^{-5} \mathrm{~m}^{2} \mathrm{~s}^{-1}$. These data yield $\Theta \approx 1$. If the liquid water content is $10 \%$ of the total water content, then $T_{0} \approx 5 \mathrm{~K}$ : that is, the droplet size is very sensitive to changes of temperature. If the droplets are of size $a=10 \mu \mathrm{m}$ and density $n_{0}=4 \times 10^{8} \mathrm{~m}^{-3}$, (which are typical values for clouds) the equilibration time is $\tau_{\text {eq }}=1.6 \mathrm{~s}$.

Ripening in a turbulent convection cell. - Now consider the response of the aerosol to convective motion in the cell. This is a consequence of how the temperature changes along the trajectories of the aerosol droplets (which are assumed to be advected by the flow).

Turbulent convection in a Rayleigh-Bénard cell is reviewed in [18 20. The upper and lower plates are at temperatures $T_{\text {up }}$ and $T_{\text {low }}$ respectively. The expectation value of the temperature is close to $T_{\mathrm{av}}=\left(T_{\mathrm{up}}+T_{\text {low }}\right) / 2$ (with small logarithmic corrections) [21, except in the vicinity of the upper and lower plates, and at any time most of the gas in the convection cell is at a temperature close to $T_{\mathrm{av}}$. Gas which is in contact with the lower plate of the cell is heated to a higher temperature $T_{\mathrm{av}}+\Delta T$ (where $\Delta T \leq \Delta T_{h} / 2$ ), and joins a plume of rising gas. The plumes persist on a timescale $\tau_{\mathrm{c}}$, which cannot exceed that of the largest eddies, $\tau_{h}=\left(h^{2} / \epsilon\right)^{1 / 3}$. It will be assumed that $\tau_{\mathrm{c}} / \tau_{\mathrm{eq}} \gg 1$. However, the mixing process is highly discontinuous. The plumes form fronts and later tendrils of approximately homogenous gas, which remain at a temperature close to the temperature that they had upon separation from the top or bottom plate until the last stage of the mixing process. In the final stage of mixing a tendril formed by the plume mixes rapidly with gas from the interior of the cell, which is at a temperature close to $T_{\text {av }}$. The final stage of mixing occurs on a much shorter timescale, namely the Kolmogorov timescale, $\tau_{\mathrm{K}}=\sqrt{\nu / \epsilon}$. The equilibration timescale will be assumed to lie between the timescales describing the flow: $\tau_{h} \gg \tau_{\text {eq }} \gg \tau_{\mathrm{K}}$.

The consequence of this picture is that droplets in the a rising plume are at a temperature, $T_{\mathrm{av}}+\Delta T$, and while 
the plume forms they equilibrate to a smaller radius,

$$
a_{1-}=a_{0}-\Lambda \Delta T, \quad \Lambda \equiv \frac{a_{0}}{T_{0}} .
$$

The gas in the plume rises, without cooling due to heat exchange, until it reaches the interior of the cell. After a timescale $\tau_{\mathrm{c}}$, the gas in the plume starts to mix with the gas in the interior. This mixing happens on a timescale which is short compared to the phase equilibration time, so that droplets of size $a_{1-}$ are mixed with the droplets in the bulk, which are of size $a_{0}$. Similarly, plumes of cold gas which form on the upper plate at a temperature $T_{\mathrm{av}}-\Delta T$ inject larger droplets, of radius $a_{1+}=a_{0}+\Lambda \Delta T$, when they fall into the bulk. The final stage of this equilibration happens on a timescale of the Kolmogorov time, which is small compared to the time required for aerosol droplets to come into equilibrium. It follows that while the temperature fluctuations associated with the plume are dissipated, fluctuations in the droplet size remain 'frozen in', resulting in a broadening of the droplet size distribution.

Now consider how this model is used to model the evolution of the droplet size distribution, $P(a, t)$. The plumes carrying gas away from the lower plate have a distribution of temperature $\Delta T$. Let $J(\Delta T) \mathrm{d} \Delta T$ be the volume of gas per unit area, per unit time, which rises from the lower plate and which has a temperature change in the interval $[\Delta T, \Delta T+\mathrm{d} \Delta T]$. It is assumed that the flux from the upper plate may be described by the same function $J(\Delta T)$. The material in this temperature range occupies a volume fraction of the gas in the column equal to

$$
\frac{\mathrm{d} V}{V}=\frac{J(\Delta T)}{h} \mathrm{~d} \Delta T .
$$

The droplets in this volume fraction undergo a change of radius equal to $\Delta a=-\Lambda \Delta T$. This results in a change of the droplet size distribution which satisfies

$$
\frac{\partial P}{\partial t}(a, t)=\int_{-\infty}^{\infty} \mathrm{d} a^{\prime} \mathcal{K}\left(a, a^{\prime}\right) P\left(a^{\prime}, t\right)
$$

where the kernel may be approximated by $\mathcal{K}\left(a, a^{\prime}\right)=$ $K\left(a-a^{\prime}\right)$ with

$$
K(\Delta a)=\frac{1}{\Lambda h}[J(\Delta a / \Lambda)+J(-\Delta a / \Lambda)]-\frac{1}{\tau_{\mathrm{c}}} \delta(\Delta a) .
$$

Here $\tau_{\mathrm{c}}$ is an estimate for the timescale of a convection roll:

$$
\frac{1}{\tau_{\mathrm{c}}}=\frac{2}{h} \int_{0}^{\infty} \mathrm{d} x J(x) .
$$

The time $\tau_{\mathrm{c}}$ cannot exceed the integral timescale of the flow: $\tau_{\mathrm{c}} \leq \tau_{\mathrm{h}}=\left(h^{2} / \epsilon\right)^{1 / 3}$. The initial rate of broadening of the particle size distribution can be related to the heat flux in the cell, which is

$$
Q=C_{p} \int_{0}^{\infty} \mathrm{d} \Delta T \Delta T J(\Delta T)
$$

where $C_{p}$ is the specific heat capacity. The growth of the mean of the absolute value of the size change is

$$
\langle|\Delta a|\rangle=\int_{-\infty}^{\infty} \mathrm{d} \Delta a|\Delta a| P\left(a_{0}+\Delta a, t\right)=\frac{2 \Lambda}{h C_{p}} Q t .
$$

It has been argued that the model predicts that droplets change size discontinuously, in steps with a magnitude comparable to $\delta a_{\max }=\Lambda \Delta T_{h} / 2$. The typical timescale separating these jumps of the particle size is $\tau_{\mathrm{c}} \leq \tau_{h}$, but the separation of the steps is random, and some droplets may experience several jump events in quick succession.

The production of rain from clouds depends upon droplets reaching a size which is significantly larger than their original size. In the context of the Rayleigh-Bénard model, this would require a droplet to undergo repeated encounters with the cold plate. Equation (9) implies that the change in surface area of a droplet is independent of its size. This implies that the growth in the particle radius after $N$ successive encounters with the upper plate satisfies

$$
\left\langle a_{N}^{2}\right\rangle=a_{0}^{2}+N \Delta A
$$

for some constant $\Delta A$.

The problem of warm rain initiation. - A cloud contains water droplets formed by condensation onto microscopic nuclei such as salt granules, dust grains, or particles of organic matter. Their concentration and droplet radius are quite variable, but the remainder of this paper uses the following representative values for a convecting cumulus cloud which could produce precipitation. The typical droplet radius is $a_{0}=10 \mu \mathrm{m}$, the number density is $n_{0}=4 \times 10^{8} \mathrm{~m}^{-3}$, and the cloud depth is $h=10^{3} \mathrm{~m}$. The rate of decrease of temperature with height (lapse rate) is $3^{\circ} \mathrm{C}$ per $1000 \mathrm{ft}$, which exceeds the adiabatic lapse rate by $1^{\circ} \mathrm{C}$ per $1000 \mathrm{ft}$, so that the effective temperature difference between the top and bottom of the cloud is $3 \mathrm{~K}$. The typical vertical velocity of air inside the cloud has magnitude $2 \mathrm{~m} \mathrm{~s}^{-1}$, so that the eddy turnover time may be taken to be $\tau_{h}=10^{3} \mathrm{~s}$. An estimate for the rate of dissipation is $\epsilon \approx h^{2} / \tau_{h}^{3}=10^{-3} \mathrm{~m}^{2} \mathrm{~s}^{-3}$, which gives an estimate of the Kolmogorov time $\tau_{\mathrm{K}} \approx 10^{-1} \mathrm{~s}$. Rain falls as droplets of size approximately $a=1 \mathrm{~mm}$. A rate of rainfall of $3.6 \mathrm{~mm} \mathrm{hr}^{-1}=10^{-6} \mathrm{~m} \mathrm{~s}^{-1}$ is described as 'moderate to heavy rainfall'.

The collision efficiencies $\varepsilon$ of small droplets are somewhat uncertain, but it is widely accepted that they are low 1,2. If the larger droplet has radius below $20 \mu \mathrm{m}$, it is believed that $\varepsilon \leq 0.1$, and that for radius $10 \mu \mathrm{m}$, $\varepsilon \leq 0.03$ 2. For droplets of size $a=50 \mu \mathrm{m}$ colliding with droplets of size $a=10 \mu \mathrm{m}$, however, the efficiencies are expected to be close to unity [1,2].

Collisions between droplets settling at a different rate yield a very small collision rate. The Stokes law for the drag on a sphere at low Reynolds number indicates that the gravitational settling rate is

$$
v=\kappa a^{2}, \quad \kappa=\frac{2}{9} \frac{\rho_{\mathrm{l}}}{\rho_{\mathrm{g}}} \frac{g}{\nu} .
$$


Inserting values for air and water at $5^{\circ} \mathrm{C}$ gives $\kappa \approx 1.4 \times$ $10^{8} \mathrm{~m}^{-1} \mathrm{~s}^{-1}$. The collision rate of a droplet of radius $a+\Delta a$ with a gas of particles of radius $a$ is

$$
\mathcal{R}=4 \pi \varepsilon n_{0} a^{2} \kappa\left[(a+\Delta a)^{2}-a^{2}\right] \sim 8 \pi \kappa \varepsilon n_{0} a^{3} \Delta a
$$

where $\varepsilon$ is the collision efficiency. Setting $\Delta a=2.5 \mu \mathrm{m}$ and $\varepsilon=0.03$ in addition to the parameters defined above gives $\mathcal{R} \approx 10^{-4} \mathrm{~S}^{-1}$. The rate of coalescence of typical sized water droplets due to collisions is therefore very small. Given that multiple collision events are required to grow a droplet to the size where runaway growth is possible, explaining growth by collisions is challenging.

Saffman and Turner 22 investigated the role of turbulence in facilitating collisions between water droplets. In the case of very small droplets, the collision rate due to turbulence is a consequence of shearing motion, so that the collision speed is of order $a_{0} / \tau_{\mathrm{K}}$. They argue that the corresponding collision rate is

$$
\mathcal{R}_{\text {turb }}=\sqrt{\frac{8 \pi}{15}} \frac{n_{0} \varepsilon(2 a)^{3}}{\tau_{\mathrm{K}}} .
$$

For the parameters of the cloud model, the gives $\mathcal{R}_{\text {turb }} \approx$ $2 \times 10^{-6} \mathrm{~s}^{-1}$, which is negligible.

After a droplet has grown to a size where it is much larger than the typical droplets, and where the collision efficiency is approximately unity, it falls rapidly and collects other droplets in its path. Consider a droplet of size $a_{1}$ falling through a 'gas' of small droplets, which can be characterised by the liquid volume fraction $\Phi_{1}=4 \pi n_{0}\left\langle a^{3}\right\rangle / 3$. The large droplet falls with velocity $v=\kappa a_{1}^{2}$ and grows in volume at a rate $\pi a_{1}^{2} \Phi_{1} v$, so that

$$
\frac{\mathrm{d} a_{1}}{\mathrm{~d} t}=\frac{\kappa \Phi_{1} a_{1}^{2}}{4} .
$$

Solving this equation shows that the droplet radius diverges in the time

$$
\tau_{\exp }=\frac{4}{\kappa \Phi_{1} a_{1}} .
$$

For the model parameters, a droplet of size $a_{1}=50 \mu \mathrm{m}$ requires time $\tau_{\exp } \approx 2 \times 10^{3}$ s to undergo explosive growth. According to (31) the time before runaway growth is expected to occur increases rapidly as the droplet size gets smaller, and this estimate must be a lower bound because it ignores the effects of collision efficiency and the settling velocity of the smaller droplets.

Rare events and rain initiation. - Consider the rate at which droplets must reach the size threshold for runaway growth. Rainfall at a rate of $3.6 \mathrm{~mm} \mathrm{hr}^{-1}=$ $10^{-6} \mathrm{~m} \mathrm{~s}^{-1}$ is considered as 'moderate'. If the raindrops have size $a \approx 1 \mathrm{~mm}$, this corresponds to raindrops falling at a rate of approximately $250 \mathrm{~m}^{-2} \mathrm{~s}^{-1}$. Given the assumed cloud depth of $h=10^{3} \mathrm{~m}$, the volumetric rate of production of raindrops is approximately $0.25 \mathrm{~m}^{-3} \mathrm{~s}^{-1}$. If the microscopic droplets have density $n_{0}=4 \times 10^{8} \mathrm{~m}^{-3}$, then the rate of conversion of each microscopic droplet into a 'collector' droplet undergoing runaway growth is approximately $6 \times 10^{-10} \mathrm{~s}^{-1}$. An alternative statement is that if a shower lasts for a five minutes, the probability that any given water droplet has grown to become a rain droplet is small, approximately $2 \times 10^{-7}$. The problem of rain initiation is, therefore, concerned with the frequency of very rare events. This point has also been made by Kostinski and Shaw [5].

Growth of droplets from the typical size of $10 \mu \mathrm{m}$ to $50 \mu \mathrm{m}$ (which is the threshold for runaway) could in principle occur by collision and coalescence. However, despite the fact that the required conversion probability is very small (of order $10^{-7}$ ), this is not achievable by a collisional mechanism. On growing from $10 \mu \mathrm{m}$ to $50 \mu \mathrm{m}$, the volume of a droplet increases by a factor of 125 , that is, there are or order 100 collision events. The conversion of a droplet to become a collector droplet requires a sequence of successive collisions which may be assumed to be statistically independent. If the rates for successive collisions were all equal to $\mathcal{R}$, the probability for $N$ collisions occurring after a short time $t$ would be

$$
P_{N} \sim \frac{(\mathcal{R} t)^{N}}{N !}
$$

It was argued above that the rate for the first collision events is small, $\mathcal{R}_{0} \approx 10^{-4} \mathrm{~s}^{-1}$. Even allowing for the fact that the collision rates increase as the droplet grows, the probability for the obtaining 100 collisions after $t=10^{3} \mathrm{~s}$ will be much smaller than $10^{-7}$. The collisional mechanism for bridging the bottleneck to runaway growth is, therefore, highly problematic.

Fast droplet growth by the convective mechanism. - As well as the theoretical difficulties of explaining droplet growth by collisional processes, observational evidence is difficult to reconcile with a collisional mechanism. Clouds may exist for long periods, before quite suddenly producing rainfall. The rapid onset of rainfall is usually associated with convective instability, which (because of the large Reynolds number) implies turbulent motion. Equation (29) indicates that the role of turbulence in facilitating particle collisions is negligible for the small droplets in the model treated here, implying that the rainfall is triggered by some other aspect of the convective process. If a parcel of air is lifted by convection, condensation occurs as the temperature falls. However, the fractional increase of the droplet size which can be achieved is not large enough to start runaway growth, and increasing the size of droplets by condensation also reduces the dispersion of their radius.

For these reasons it is desirable to find other mechanisms whereby convection can result in runaway growth. The Rayleigh-Bénard cell can serve as a model for convective motion in a cloud, and it will be argued that the convective ripening mechanism can result droplet growth rates which are more rapid than the collisional mechanism. 
Droplets can grow or shrink due to changes in the level of supersaturation. Consider the circulation of a droplet in a rising packet of air. This air mass is cooled slowly (on a timescale $\tau_{\mathrm{c}} \gg \tau_{\text {eq }}$ ) by radiation when it reaches the top of the cloud. The droplets that it contains increase in size by condensation, due to capacity of the surrounding air to carry water vapour being reduced. The cool packet of air then becomes part of a 'plume' of descending air, which falls far into the body of the cloud before being rapidly mixed with ambient air (on a timescale $\tau_{\mathrm{K}} \ll \tau_{\text {eq }}$ ). The temperature of the descending plume increases due to adiabatic compression as the air pressure rises, but it is still colder than the surrounding gas at the time when mixing occurs. The mixing occurs so rapidly that the droplets are unable to evaporate, and their increased sizes are 'frozen'. If the droplets are close to the condensation level (the base of the cloud) at the time when mixing occurs, the droplets will achieve a size which is much larger than the surrounding droplets. One single cycle of this process is not sufficient to bridge the bottleneck and achieve runaway growth. It will be argued that, compared to collisional processes, this mechanism can require fewer steps for droplets to grow to the size where runaway growth is possible, and that these steps can occur in a shorter timescale. This mechanism requires $\tau_{\mathrm{c}} \gg \tau_{\mathrm{eq}} \gg \tau_{\mathrm{K}}$. These inequalities are easily satisfied for the model cloud parameters, where $\tau_{\mathrm{c}} \approx \tau_{h} \approx 10^{3} \mathrm{~s}, \tau_{\mathrm{eq}} \approx 1 \mathrm{~s}$ and $\tau_{\mathrm{K}} \approx 10^{-2} \mathrm{~s}$.

Because $\tau_{\mathrm{c}}^{-1}$ is larger than the rate of collision of small particles, the steps of the convective ripening mechanism are more frequent than those of the collisional process. Recall that raindrops grow from a very small number of microscopic droplets: in this case the shower is triggered by droplets which happen to be convected to the upper surface of the cloud several times in quick succession.

Equation (9) implies that growth of water droplets by condensation increases the area of a droplet by an amount which is independent of the droplet size on each cycle. Consider what happens as a droplet makes repeated encounters with the top of a cloud, followed by rapid warming in the interior. Under the most favourable circumstances, where a droplet falls repeatedly falls very close to the base of the cloud before its surroundings are mixed with the ambient air, the number of cycles required to increase the radius from $10 \mu \mathrm{m}$ to $50 \mu \mathrm{m}$ is $(50 / 10)^{2}=25$. This is much smaller than the number of events required for the collisional growth, which is $(50 / 10)^{3} \approx 125$, and the timescale separating each event is shorter.

Concluding remarks. - This paper has described a non-collisional model for increasing the dispersion of droplet sizes in a Rayleigh-Bénard cell. This is used as a model for resolving the droplet growth bottleneck problem in cloud physics. It has been argued above that the dominant mechanism for creating larger droplets is that droplets grow slowly by condensation as they are convected upwards in a cloud, but that the increased size is frozen in when a falling plume of cold air is mixed rapidly in the interior of the cloud.

The convective ripening mechanism was compared with collisional growth in clouds. The convective ripening mechanism discussed here can bridge the growth bottleneck in fewer steps, which occur at a higher rate. Further work in required to quantify the statistics of repeated contacts with the cool plate of the convection cell, because this determines the rate at which the largest droplets can grow.

Acknowledgements. This paper was written with the generous support of the NORDITA program 'Dynamics of Particles in Flows'.

\section{REFERENCES}

[1] Mason, B. J., The Physics of Clouds (University Press, Oxford) 1957

[2] H. R. Pruppacher H. R. and Klett, J. D., Microphysics of Clouds and Precipitation, 2nd ed. (Kluwer,Dordrecht) 1997

[3] Rogers, R. R. And Yau, M. K., A Short Course in Cloud Physics (Pergamon, Oxford) 1982

[4] Shaw, R. A., Ann. Rev. Fluid Mech., 35 (2003) 183-227

[5] Kostinski, A. B. and Shaw, R. A., Bull. Am. Met. Soc., 86 (2005) 235-44

[6] Lifshitz, E. M. and Slyozov, V. V., J. Phys. Chem. Solids, 19 (1961) 35

[7] Clement, C. F., Environmental chemistry of aerosols (Blackwell Publishing, Oxford) 2008, p. 49-89

[8] Wilkinson, M., Europhys. Lett., 106 (2014) 40001

[9] Brenguier, J.-L. and Chaumat, L., J. Atmos. Sci., 58 (2001) 628

[10] Chaumat. L. and Brenguier, J.-L., J. Atmos. Sci., 58 (2001) 642

[11] Vaillancourt, P. A., Yau M. K. and Grabowski, W. W., J. Atmos. Sci., 58 (2001) 1945

[12] Vaillancourt, P. A., Yau M. K., Bartello, P. and Grabowski, W. W., J. Atmos. Sci., 59 (2002) 3421

[13] Andrejczak, M., Grabowski, W. W., Malinowski, S. P. And Smolarkiewicz, , J. Atmos. Sci., 61 (2004) 1726

[14] Celani, Falkovich, G., Mazzino, A., and Seminara, A., Europhys. Lett., 70 (2005) 775

[15] Sidin, R. S. R., IJzermans, R. H. A. and Reeks, M. W., Phys. Fluids, 21 (2009) 106603

[16] Lanotte, A. S., Seiminara, A. and Toschi, F., J. Atmos. Sci., 66 (2009) 1685

[17] Kumar, B., Schumacher, J. and Shaw, R. A., Theor. Comput. Fluid Dyn., 27 (2013) 361-76

[18] Siggia, E. D., Ann. Rev. Fluid Mech., 26 (137) 1994

[19] Bodenscahtz, E., Pesch, W. and Ahlers, G., Ann. Rev. Fluid Mech., 32 (709) 2000

[20] Ahlers, G., Grossmann, S. and Lohse, D., Rev. Mod. Phys., 81 (503) 2009

[21] Ahlers, G., Bodenschatz, E., Funfschilling, D., Grossmann, S., He, X., Lohse, D., Stevens, R. J. A. M. and Verzicco, R., Phys. Rev. Lett., 109 (2012) 114501

[22] Saffman, P. G. and Turner, J. S., J. Fluid. Mech., 1 (16-30) 1956 